\title{
Positron annihilation in boron nitride
}

\author{
N.Amrane \\ United Arab Emirates University Faculty of Science, Physics Department, \\ Al Ain P.O.Box17551 United Arab Emirates
}

Received January 24, 2006, in final form May 20, 2006

\begin{abstract}
Electron and positron charge densities are calculated as a function of position in the unit cell for boron nitride. Wave functions are derived from pseudopotential band structure calculations and the independent particle approximation (IPM), respectively, for electrons and positrons. It is observed that the positron density is maximum in the open interstices and is excluded not only from ion cores but also to a considerable degree from valence bonds. Electron-positron momentum densities are calculated for $(001,110)$ planes. The results are used in order to analyse the positron effects in BN.
\end{abstract}

Key words: positron annihilation, momentum density, angular correlation

PACS: 78.70.Bj, 71.15.Dx

\section{Introduction}

Boron nitride can be synthesized in hexagonal (h-BN) or in cubic form (c-BN), according to the growth conditions. Both of them present numerous attractive properties, such as high chemical inertness, low density, wide range of transparency, and good thermal conductivity. However, the hexagonal form is mechanically softer than the cubic phase, and above all is anisotropic. This anisotropy is evidenced in all its physical properties, such as mechanical strength, electrical resistivity, thermal conductivity [1] or optical indices [2]. Therefore, in thin films growth, the control or the determination of the $c$-axis orientation is of major importance. In the publications it is reported that this axis can be oriented in the plane of the growing film as well as in all the directions up to the normal of the sample. This orientation can be determined by interpreting the infrared transmittance spectroscopy measurements performed at non-normal incidence, based on the optical model of an uniaxial medium developed by Schubert et al. [2].

Boron nitride is a wide band-gap (gap $\sim 5.8 \mathrm{eV}$ ) III-nitride semiconductor which is transparent in a wide range of wavelengths, from the ultraviolet to the infrared region. It has been widely used as a coating material for tribological applications and as the passivation layer and insulating dielectric in microelectronic devices. In fact, since a piezoelectric surface layer like $\mathrm{AlN}$ or $\mathrm{ZnO}$ is often required to generate surface acoustic waves, h-BN belonging to the $6 \mathrm{~mm}$ symmetry class can be an excellent substrate for acousto-optic devices. Its high velocity of sound suggests possible applications in surface acoustic wave devices (SAW).

Most of all, its low chemical re-activity with transition-metal alloys makes it an unequalled substitute to diamond for the machining of ferrous materials [3-6]. So, the synthesis of cubic boron nitride has been motivated.

It is well known that cubic boron nitride can be synthesized under high pressures and high temperatures from the graphite-like hexagonal modification $(\mathrm{hBN})$ or in the pressure of a suitable catalyst-solvent. However, even with the use of catalysts, the pressure is higher than 4.0 GPa [7-9]. Besides, it has been found that cBN formation could be obtained at pressures down to 2.5 GPa using amorphous boron nitride as starting materials [10]. Recently, Hao et al. reported that cBN was synthesized at low pressure.

For the past few years, the ease of fabrication of this material using new processes such as plasma enhanced chemical vapor deposition (PECVD), reactive ion plating (RIP), sputtering, ion 
beam deposition techniques [11,12] has opened a large area of applications. Materials such as $\mathrm{ZnO}$, AlN and sputtered LiNbO3 have been widely investigated for a long time.

Recently several calculations were done for the ground-state properties of both cBN and hBN [13-15]. The present study extends these investigations of the electronic structure of BN using positrons.

The investigation of the electronic structure of solids using positrons occupies a place of increasing importance in solid state physics [16,17]. The recent growth in positron studies of defect trapping in semiconductors [18-21] suggests the desirability of an improved theoretical understanding of the annihilation parameters for such systems.

Although there has been some attempt to study the behavior of the positron wave function in compound semiconductors [22-25], so far no calculations have been reported on the angular correlation of positron annihilation radiation (ACPAR) lineshapes for BN. This has prompted us to take up such calculations.

The theoretical calculations of the lineshapes are carried out employing a pseudopotential band model for computation of the electron wave function. The positron wave function is evaluated under the point core approximation (the independent particle model). The crystal potential experienced by a positron differs from that experienced by an electron. Since we assume that there is at most one positron in the crystal at any time, there are no positron-positron interactions, i.e., exchange or corrections. Thus positron potential is partly caused by the nuclei and partly by the electrons, both components being purely coulombic in nature.

The density functional theory (DFT) combined with the local density approximation (LDA) or with the generalized gradient approximation (GGA) [26-28] is one of the most efficient techniques of electron-structure calculations. It has also been used for positron states in bulk metals in order to determine the momentum distribution of the annihilating positron-electron pairs [29]. However, those calculations are technically difficult and computationally time consuming. It is well known that electronic structure based on the DFT calculations underestimates the band gaps by as much as $50-100 \%$. The LDA, also overestimates the positron annihilation rate in the low-momentum regime, thus giving rise to positron lifetimes shorter than the experimental values. Moreover, the LDA overestimates the cohesive energy in electronic structure calculations, for reasons connected with the shape of the correlation hole close to the nucleus. The empirical methods [30-32], while simple in nature, and with the drawback that a large number of fitting parameters are required, are very accurate and produce electronic and positronic wave functions that are in good agreement with experiments. This approach was encouraged by the work of Jarlborg et al. who discovered that the empirical pseudopotentials gave a better agreement with the experimental electronic structures than the first-principles calculations [33].

We remark, at this point, that while a positron in a solid state is a part of the system with important many-body interactions, the quantum independent model (IPM) is often very useful. Positron annihilation techniques have resulted in very useful information on the electron behavior in semiconductors and alloys. The initially large energy positron $(1 \mathrm{MeV})$ rapidly loses energy in the sample mostly through ionization and excitation processes, when the positron is in thermal equilibrium with the sample, and annihilation occurs with a valence electron yielding two $\gamma$ rays. The positron lifetime measurements yield information [34] on the electron density at the position of the positron. Moreover, the angular correlation of the two $\gamma$-rays resulting from the most probable decay process can be measured. The two photons arising from the annihilation are nearly collinear due to the conservation of momentum. Since these photons are created by positron annihilation with electrons in a solid and the momentum distribution of the photons thus corresponds to that of the electrons, this provides information on the momentum distribution of the annihilating positronelectron pair. There have been experimental investigations on several semiconductors, such as GaN, AlN [18]. This work provides complementary theoretical data to show the power of the independent particle approximation.

In the case of metals or alloys, the LCW folding theorem [35] applied to the positron annihilation is well known to give a powerful means of sampling the occupied states and gives direct information of the geometry of the Fermi surfaces. For semiconductors, however, it is not clear what kind of 
information could be obtained. One may expect by analogy with metals to obtain the geometry of the occupied $k$-space, namely the first Brillouin zone. Experimental results in this approach are not yet reported for semiconductors. In order to investigate the electronic states of bonds, we applied the LCW theorem to the positron annihilation. The details of calculations are described in section 2 of the present paper. The results for BN are discussed in section 3.

\section{Calculations}

The electron and positron wave functions are essential ingredients in the calculation of the electron-positron $k$-space densities. We therefore focus our attention on the evaluation of electron wave function derived from band structure calculations. One of the central problems in the band theory of solids is to find the propagating solution of a Schrodinger equation

$$
\left\{\frac{p^{2}}{2 m}+V(r)\right\} \psi_{n k}(r)=E_{n k} \psi_{n k}(r)
$$

in which the potential has the periodicity of the lattice. Exact solutions of this problem are in general not possible, and so a number of approximation methods have been used so far.

In our case, we have used the empirical pseudo-potential method (EPM), which involves a direct fit of the atomic form factors $\mathrm{V}(\mathrm{G})$ to the experimental band structure. Therefore, the first step in this calculation is to choose the best possible set of form factors, which will allow us to obtain the theoretical band structure. The experimentally known energy gaps at $\Gamma, \mathrm{X}$ and $\mathrm{L}$ points of the Brillouin zone are taken as a reference.

Let us define our empirical pseudo-potential parameters (EPP) of a semiconductor as a superposition of the pseudo-atomic potential of the form $V(r)=V_{\mathrm{L}}(r)+V_{\mathrm{NL}}(r)$, where $V_{\mathrm{L}}$ and $V_{\mathrm{NL}}$ are local and nonlocal parts, respectively. In these calculations we have omitted the nonlocal part. We regard the Fourier components of $V_{\mathrm{L}}(r)$ as the EPP local parameters.

We determine the EPP parameters by a nonlinear least squares method, in which all the parameters are simultaneously optimized under a defined criterion of minimizing the root-mean square (rms) deviation. The experimental electronic band structure data are used at normal pressure.

Our nonlinear least squares method requires that the rms deviation of the calculated level spacing (LS) from the experimental ones defined by

$$
\delta=\left[\frac{\sum\left(\Delta E^{(i, j)}\right)^{2}}{m-N}\right]^{1 / 2}
$$

should be minimum.

$$
\Delta E^{(i, j)}=E_{\text {exp }}^{(i, j)}-E_{\text {calc }}^{(i, j)}
$$

where $E_{\text {exp }}^{(i, j)}$ and $E_{\text {calc }}^{(i, j)}$ are the observed and calculated LSs between the $i$-th state at the wave vector $\boldsymbol{k}=\boldsymbol{k}_{\mathrm{i}}$ and the $\boldsymbol{j}$-th state at $\boldsymbol{k}=\boldsymbol{k}_{j}$, respectively, in the m chosen pairs $(\mathrm{i}, \mathrm{j})$. $\mathrm{N}$ is the number of the EPP parameters. The calculated energies given by solving the EPP secular equation depend nonlinearly on the EPP parameters.

The valence electron density $\rho(r)$ is defined as

$$
\rho_{\mathrm{e}}(r)=2 \sum_{n} \sum_{k}\left|\Psi_{n k}(r)\right|^{2},
$$

where $\Psi_{n k}$ is the wave function of the valence electron with the wave vector $k$ in the $n$-th valence band. The summations are taken over the occupied states (we have used about 1200 $\boldsymbol{k}$-points).

The pseudopotential method used here starts with that used by Bergstresser and Cohen in their well known treatment of cubic binary compounds [36]. The pseudopotential Hamiltonian contains 
an effective potential which is expanded as Fourier series in a reciprocal lattice space. For a binary compound the expansion is written in two parts which are symmetric and antisymmetric with respect to an interchange of two atoms about their midpoint:

$$
V(r)=\sum_{G}\left[S^{S}(G) V_{G}^{S}+S^{A}(G) V_{G}^{A}\right] \exp (\mathrm{i} G . r)
$$

$\mathrm{G}$ are the reciprocal lattice vectors. The structure and form factors are given by:

$$
\begin{gathered}
S^{S}(G)=\cos G . \tau, \quad S^{A}(G)=\sin G . \tau \\
V_{G}^{S, A}(1,2)=\frac{1}{\Omega_{1,2}} \int \frac{1}{2}\left[V_{1}(r) \mp V_{2}(r)\right]_{1,2} \exp (-\mathrm{i} G . r) \mathrm{d}^{3} r .
\end{gathered}
$$

Here $\tau=\mathrm{a} / 8(111)$ is half the vector between the two atoms contained in the unit cell, a is the lattice parameter and $V_{G}^{S, A}(1,2)$ are the pseudopotential form factors of the individual atoms.

This can be simplified through the use of transferability approximation for atomic pseudopotentials as discussed by Phillips [37], by writing:

$$
V_{G}(J)=\frac{1}{\Omega_{j}} \int V_{\mathrm{i}}(r) \exp (-\mathrm{i} G \cdot r) \mathrm{d}^{3} r
$$

where $\Omega_{j}$ is the volume per atom of the monoatomic solid consisting of atoms of type $j$.

The charge density is $e \rho(r)$, where $e$ is the electron charge. This has the Fourier transform given by

$$
\rho(G)=\frac{1}{\Omega} \int \rho(r) e^{\mathrm{i} G r} \mathrm{~d}^{3} r
$$

We follow the approach of Aourag et al. [23] to evaluate the positron wave function; the total positron potential can be expressed as

$$
V_{p}(r)=V_{\mathrm{i}}(r)+V_{\mathrm{c}}(r)+V_{\mathrm{ep}}(r)
$$

where $V_{\mathrm{i}}(r), V_{\mathrm{c}}(r)$, and $V_{\mathrm{ep}}(r)$ are the ionic, Coulomb, and electron-positron correlation potentials, respectively.

Since $V_{\mathrm{i}}(r)$ is a periodic potential and the zincblende structure involves non-primitive lattice translations, it is expressed as

$$
V_{\mathrm{i}}(r)=\sum \sum v_{\mathrm{i}}\left(r-R_{n}-T_{\mathrm{i}}\right),
$$

where $\mathrm{R}_{n}$ denotes the set of all Bravais lattices vectors, it consists of all points with position vectors $\mathrm{R}_{n}$, where the $n$ 's range all through positive and negative integer values and $\mathrm{T}_{\mathrm{i}}$ is a non-primitive vector of a two-atom basis. The set of vectors $\mathrm{T}$ describes the so called translational symmetry of a lattice.

The nuclear charge can be expressed as a $\delta$-function at the origin of each cell and in the core approximation:

$$
v_{\mathrm{i}}(\mathbf{r})=\frac{Z e^{2}}{\mathbf{r}}
$$

On the other hand, the electron-positron coulomb potential is expressed as:

$$
V_{\mathrm{c}}(\mathbf{r})=-2 \int \frac{\rho_{\mathrm{e}}\left(r^{\prime}\right) \mathrm{d}^{3} r}{\left|r-r^{\prime}\right|},
$$

where $\rho_{\mathrm{e}}(r)$ is the charge density of the valence electrons for the binary semiconductor. $\rho_{\mathrm{e}}(r)$ has been calculated using the empirical pseudopotential scheme (EPM). The electron-positron potential is a slow function of the electron density (only one positron). It is generally flat in the interstitial region and swamped by the $\mathrm{V}_{\mathrm{i}}(\mathrm{r})$ and $\mathrm{V}_{\mathrm{c}}(\mathrm{r})$ in the ion core region. Hence it is not considered here. This gives good results. 
The positron density is evaluated by using $\Psi_{n k}(r)$ as in equation (4). In our calculations, a fully thermalized positron is assumed to be, in good approximation, at the bottom of the positron band with $k=0$ and $n=1$

$$
\rho_{p}(r)=\left|\Phi_{n=1, k=0}\right|^{2}
$$

The EPM scheme employs an extended plane wave basis, where the pseudo-wave function is expressed as an expansion over an arbitrary large number of plane waves,

$$
\Psi_{n k}(r)=\sum_{G} \psi_{n}(k+G) e^{\mathrm{i}(k+G) \cdot r}
$$

In our calculations the expansion is cut off at $14 \mathrm{Ry}$, thus including 136 plane waves.

The wave function of the thermalized positron in the reciprocal space is given by:

$$
\Psi_{+}(r)=\frac{1}{\sqrt{\Omega}} \sum A(G) e^{\mathrm{i} G r}
$$

The coefficients $\mathrm{A}(\mathbf{G})$ are found out by solving the secular equation for the positron. The positron can be described by a band model with one positron per unit cell independent-particle model (IPM). The probability of annihilating the $e^{-}-e^{+}$pair with momentum $\mathbf{p}$ is proportional to the pair momentum density:

$$
\rho^{2 \gamma}(p)=\sum_{n, k} \eta_{n}(k)\left|\int \mathrm{d}^{3} r e^{-\mathrm{i} p r} \Psi_{n, k}(r) . \Psi_{+}(r)\right|^{2},
$$

where $\Psi_{n, k}(r)$ and $\Psi_{+}(r)$ are the electron and positron Block wave functions and $\eta_{n}(k)$ is the occupation number. In the long-slit angular correlation experiment one measures a component of the pair momentum density as given by

$$
N\left(p_{z}\right)=\iint \rho^{2 \gamma}(p) \mathrm{d} p_{x} \mathrm{~d} p_{y}
$$

It is usual to perform a "Lock-Crisp-West" (LCW) zone folding [35] of the various extended zone components of $\rho(p)$ into the first Brillouin zone, thus forming the zone-reduced momentum density:

$$
n(k)=\sum_{G_{\mathrm{i}}} \rho\left(p+G_{\mathrm{i}}\right)
$$

where $G_{\mathrm{i}}$ is the $i$-th reciprocal lattice vector defined within the first Brillouin zone. Using Block's theorem, $n(k)$ can be described as:

$$
n(k)=\text { const } \sum_{n} \theta\left(E_{\mathrm{F}}-E_{n, k}\right) \int\left|\psi_{n, k}(r) \phi(r) \mathrm{d} r\right|^{2},
$$

where $E_{\mathrm{F}}$ is the Fermi energy and $\theta\left(E_{\mathrm{F}}-E_{n, k}\right)$ is a step function as follows:

$$
\theta\left(E_{\mathrm{F}}-E_{n, k}\right)= \begin{cases}1, & E_{\mathrm{F}} \leqslant E_{n, k} \\ 0, & E_{\mathrm{F}} \geqslant E_{n, k}\end{cases}
$$

The parameters used for this calculation are listed in table 1, the calculated Fourier coefficients of the valence charge densities for $\mathrm{BN}$ are given in table 2 .

\section{Results}

In the first step of our calculations, we have computed the Fourier coefficients of the valence charge densities using the empirical pseudopotential method (EPM). This method has proved to be quite sufficient to qualitatively describe the realistic charge densities. As input, we have introduced 
Table 1. The adjusted symmetric and antisymmetric form factors (in Ry), and the lattice constant $a_{o}$ (in atomic units) for BN used in these calculations.

\begin{tabular}{|l|l|l|l|l|}
\hline compound & $\begin{array}{l}\text { Adjusted lattice } \\
\text { constant } \mathrm{a}_{o}\end{array}$ & $\begin{array}{l}\text { Experimental } \\
\text { lattice constant } \\
\mathrm{a}_{o}[42]\end{array}$ & $\begin{array}{l}\text { Adjusted form } \\
\text { factors }\end{array}$ & $\begin{array}{l}\text { Experimental form } \\
\text { factors [42] }\end{array}$ \\
\hline $\mathrm{BN}$ & 6.8333 & 6.8241 & $\mathrm{~V}_{s}(3)=-0.7706$ & $\mathrm{~V}_{s}(3)=-0.755$ \\
& & $\mathrm{~V}_{s}(8)=0.1985$ & $\mathrm{~V}_{s}(8)=0.182$ \\
& & $\mathrm{~V}_{s}(11)=0.1330$ & $\mathrm{~V}_{s}(11)=0.133$ \\
& & $\mathrm{~V}_{a}(3)=0.2912$ & $\mathrm{~V}_{a}(3)=0.1755$ \\
& & $\mathrm{~V}_{a}(4)=0.1250$ & $\mathrm{~V}_{a}(4)=0.1250$ \\
& & $\mathrm{~V}_{a}(11)=0.0251$ & $\mathrm{~V}_{a}(11)=0.0251$ \\
\hline
\end{tabular}

Table 2. The calculated Fourier coefficients of the valence charge densities for BN.

\begin{tabular}{|l|lc|}
\hline $\mathbf{G}(a / 2 \pi)$ & Fourier coefficients $(\mathrm{e} / \Omega)$ for $\mathbf{B N}$ \\
\hline 000 & 8.0000 & 0.0000 \\
111 & 0.2356 & -0.4266 \\
220 & 0.0112 & 0.0189 \\
311 & -0.0201 & -0.0431 \\
222 & 0.0000 & -0.1628 \\
400 & 0.0000 & 0.0160 \\
331 & -0.0037 & 0.0031 \\
\hline
\end{tabular}

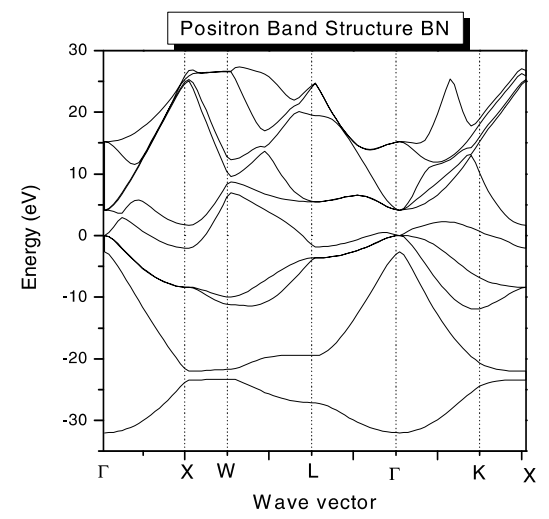

Figure 1. Positron energy band structure along principal symmetry lines for BN.

the form factors (the symmetric and antisymmetric parts) and the lattice constant for BN. The resulting Fourier coefficients are used to generate the corresponding positron wave function using the ThApositron band structure for BN is displayed in figure 1. We note the astonishing similarity to its electron counterpart, with the exception that the positron energy spectrum does not exhibit a band gap. This is consistent with the fact that these bands are all conduction bands. An oversimplified explanation of this similarity has been presented elsewhere [38], in terms of the electron and positron potential. The calculated positron charge densities in the (110) plane and along the $\langle 111\rangle$ direction are displayed in figures $(2 \mathrm{a}, 2 \mathrm{~b})$. It is seen that the positron is located in the interstitial region and that the probability is low around the positions of the nuclei. The positron is repelled by the positively charged atomic cores and tend to move in the interstitial regions. The maximum of the charge is located at the tetrahedral site. From the quantitative point of view, there is a 


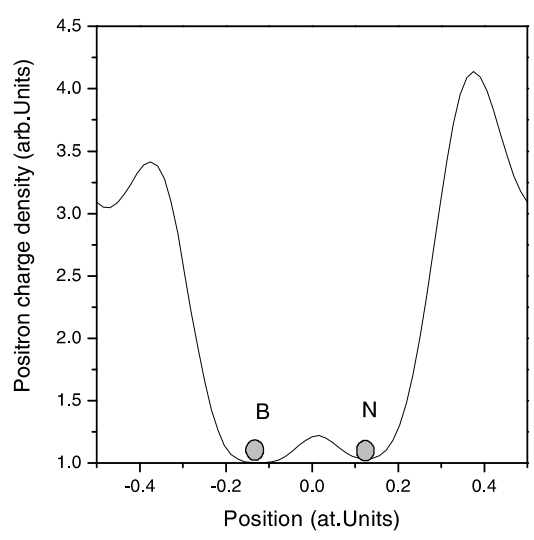

(a)

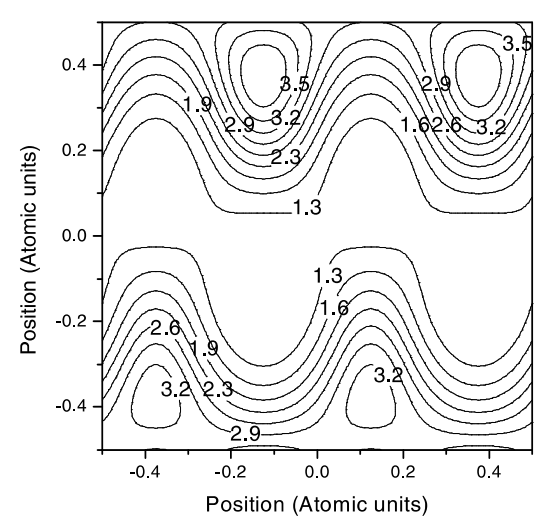

(b)

Figure 2. (a) The thermalized positron charge density in $B N$ at the $\Gamma_{1}$ point along $\langle 111\rangle$ direction. (b) The thermalized positron charge density in $B N$ at $\Gamma_{1}$ point in the (110) plane.

difference of charge in the interstitial regions, the positron distribution being more pronounced in the neighborhood of the $\mathrm{N}$ anion than in that of the $\mathrm{B}$ cation. These differences in profiles are immediately attributable to the cell which contains a larger valence and a larger ion core. We are considering the implications of this in regard to the propensity for positron trapping and the anisotropies that might be expected in the momentum densities for both free and trapped positron states. We should point out that a good agreement of the band structure and charge densities was used as an indication of both the convergence of our computational procedure and the correctness of the pseudopotential approach using the adjusted form factors. These latter as well as the lattice constant have been adjusted to the experimental data prior to the calculations.

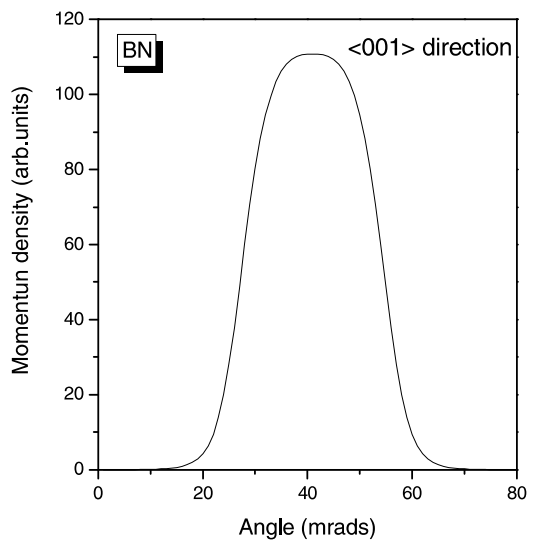

Figure 3. The integrated electron-positron momentum density in $\mathrm{BN}$ along the $\langle 001\rangle$ direction.

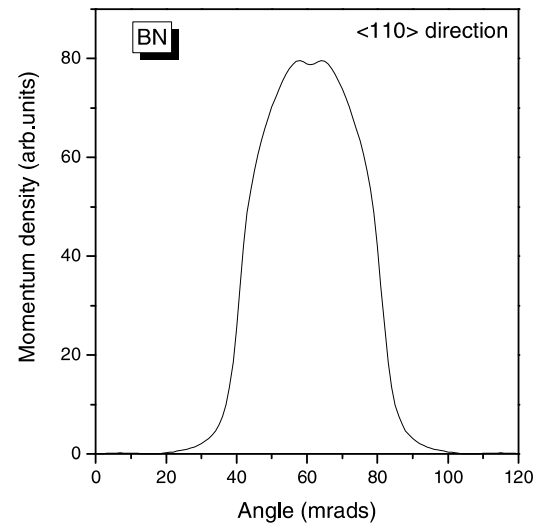

Figure 4. The integrated electron-positron momentum density in $\mathrm{BN}$ along the $\langle 110\rangle$ direction.

Let us now discuss the results of the calculated 2D-electron-positron momentum density for $\mathrm{BN}$, obtained by integration of the appropriate plane along the $\langle 110\rangle$ and $\langle 001\rangle$ directions (figures 3 and 4), the first obvious observation is that the profiles exhibit marked departures from simple inverted parabola, suggesting that for BN the electrons behave as nearly free (NFE). At the low momentum region, the profile along the $\langle 001\rangle$ direction is seen to be flat as observed in Ge and $\mathrm{Si}$ [39]. Compared to this, the profile along the $\langle 110\rangle$ direction is sharply peaked. However, the valleys and dips observed in $\rho(p)$ for BN are very shallow as compared with those of Si and Ge. This fact clearly tells us that the momentum dependence of $\rho(p)$ is very much different between elemental 
and compound semiconductors. In the case of $\mathrm{Si}$, the symmetry is $O_{h}^{7}$ which contains 48 symmetry operations including glide and screw, while in the case of BN, the symmetry is lowered from $O_{h}^{7}$ to $T_{d}^{2}$ : the two atoms in each unit cell are inequivalent and the number of symmetry operations thus decreases from 48 to 24 . Since the glide and the screw operations are not included in this space group, this crystal is symmorphic. It is emphasized that the symmetry lowering from $\mathrm{O}_{h}$ to $\mathrm{T}_{d}$ revives some of the bands which are annihilation inactive in the case of Si. If this symmetry lowering effect is large enough, the ratio in the annihilation rate of the [110] line to the [001] one becomes small since the bands become annihilation active for both ridge [110] and valley [001] lines. From the cal-

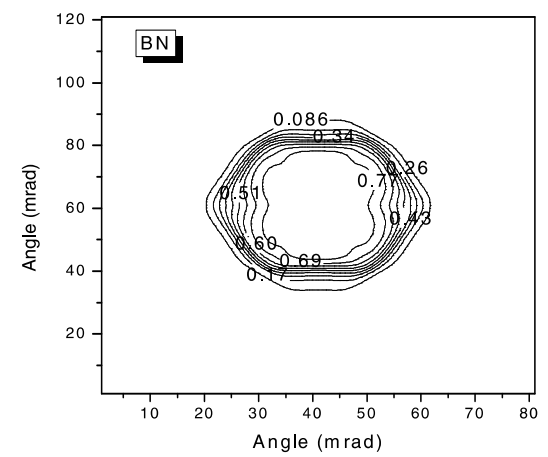

(a)

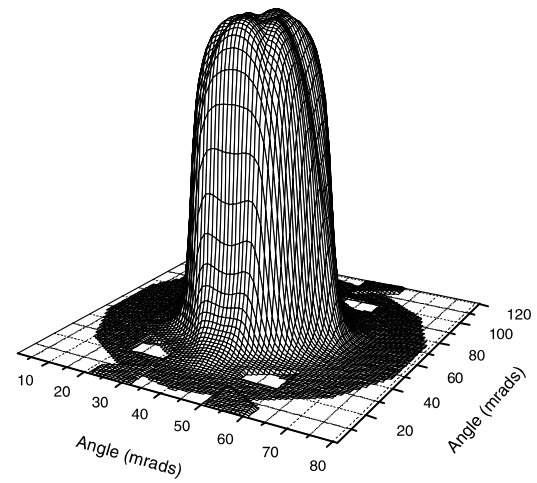

(b)

Figure 5. The calculated electron -positron momentum densities for BN in the (001-110) plane: (a) contour maps; (b) bird's eye view.

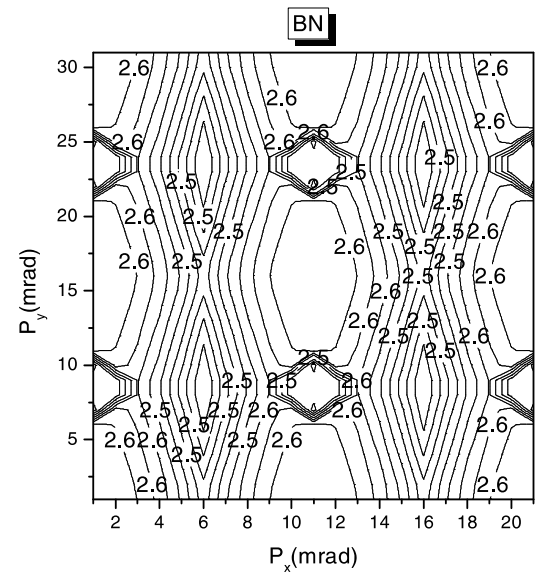

Figure 6. The calculated electron-positron momentum density after LCW folding in BN.

culations performed by Saito et al. [40] in GaAs, it was found that the contribution of these revived bands to the annihilation rate is small. The sharp peaking along the $\langle 110\rangle$ direction and the flatness of the peak along the $\langle 001\rangle$ direction could also be understood in terms of the contribution of $\sigma$ and $\pi^{*}$ orbitals to the ideal $\mathrm{sp}^{3}$ hybrid ones. Since the electronic configuration of boron is $1 \mathrm{~s}^{2} 2 \mathrm{~s}^{2} 2 \mathrm{p}^{1}$ and that of nitrogen is $1 \mathrm{~s}^{2} 2 \mathrm{~s}^{2} 2 \mathrm{p}^{3}$, the interaction between second neighbour $\sigma$ bonds is equivalent to a $\pi$ antibonding interaction between neighbouring atoms. As a consequence, there is a strong $(2 \mathrm{p}, 2 \mathrm{p}) \sigma$ bond along $\langle 110\rangle$ direction and an admixture of $(2 \mathrm{p}, 2 \mathrm{p}) \sigma$ and $(2 \mathrm{p}, 2 \mathrm{p}) \pi^{*}$ bonds along $\langle 001\rangle$ direction, the explanations are in good agreement with an earlier analysis based on group theory [16].

The calculated electron-positron momentum density (contour maps and bird's eye view of reconstructed 3D momentum space density) in the (110-001) plane is displayed in figures $5(\mathrm{a})$ and 
5(b). There is a good agreement in the qualitative feature between our results and experimental data obtained by Berko and co-workers for carbon [41], one can notice that there is a continuous contribution, i.e. there is no break, thus all the bands are full. The contribution to the electronpositron momentum density are at various $\mathbf{p}=\mathbf{k}+\mathbf{G}$. In case of elemental semiconductors like $\mathrm{Si}$, a set of bonding electrons is composed of $3 \mathrm{p}$ electrons, the distortion is expected to be observed since both of the $2 \mathrm{p}$ and $3 \mathrm{p}$ set of electrons possess a perfect point symmetry. But it can be seen that for BN, the degree of distortion is smaller than in Si. Compared to this result, the number of contour lines is smaller and the space between the contour lines is wider in BN system.

Figure 6 gives the calculated LCW folded distribution for BN. The momentum distribution in the extended zone scheme is represented by $\mathrm{n}(\mathrm{k})$ in the reduced zone scheme. We can deduce from the map that the electronic structure consists entirely of full valence bands, since the amplitude variation in the LCW folded data is merely constant.

\section{Conclusion}

In the present paper we have reported positronic distributions for BN calculated within the pseudopotential formalism and employing the independent particle model (IPM). These distributions are found to be strongly effected by the actual symmetry of the orbitals taking part in bonding. Therefore, it is expected that the positron-annihilation technique is an effective tool and a sensitive microscopic probe of semiconductors. We have shown that by performing the electron-positron momentum densities, a deep insight into the electronic properties can be achieved. More importantly, due to its relatively few assumptions, the present theory yields a reliable single-particle description of positron annihilation. Thus, it represents an excellent starting point for a systematic many-particle description of the process.

\section{References}

1. Slack G.A., J. Phys. Chem. Solids, 1973, 34, 321.

2. Schubert M., Rheinlander B., Franke E., Neumann H., Hahn J., Roder M., Richter F., Appl. Phys. Lett., 1997, 70, 1819.

3. Will G., Perkin P.G., Mater. Lett., 1999, 40, 1-4.

4. Vel L., Demazeau G., J. Etourneau, Mater. Sci. Eng. B, 1991, 10, 149-151.

5. Jiang L., Fitzgerald A.G., Rose M.J., et al., Appl. Surf. Sci., 2000, 167, 89-93.

6. Bocqillon G., Lorewrs-Susse C., J. Loriers, J. Mater. Sci., 1993, 28, 3547-3556.

7. Solozhenko V.L., Turkevich V.Z., Holzapfel W.B., J. Phys. Chem. B, 1999, 103, 8137-8140.

8. Kagamida M., Kanda H., Akaishi M., et al., J. Cryst. Growth, 1989, 94, 261-269.

9. Saito T., Endo T., Kashima S., et al., J. Mater. Sci., 1983, 18, 3054-3062.

10. Singh B.P., Solozhenko V.L., Will G., Diam. Relat. Mater., 1995, 4, 1193-1195.

11. Davis R.F., Proc. IEEE, 1991, 79, 7-11.

12. Boudiombo J., Baehr O., Boudrioua A., Thevenin P., Loulergue J.C., Bath A., Mat. Sci. Eng. B, 1997, 46, 96 .

13. Susumu Okada, Susumu Saito and Atsushi Oshiyama, Physica B, 2002, 323, 224-226.

14. Ferhat M., Zaoui A., Certier M., Aourag H., Physica B, 1998, 252, 229-236.

15. Gorczyca I., Christensen N.E., Physica B, 1994, 185, 410-414.

16. Saito M., Oshiyama A., Tanigawa S., Phys. Rev. B, 1991, 44, 10601.

17. Panda B.K., Mahapatra D.P., Padhi H.C., Phys. Stat. Sol. (b), 1992, 169, 89.

18. Arutyunov N.Yu., Emstev V.V., Mikhailin A.V., Davidov V.Yu., Physica B, 2001, 308-310, 110-113.

19. Puska M.J. et al., Phys. Rev. B, 1995, 52, 10947.

20. Lin J.N., Sun J.N., Gidley D.W., Wetzel J.T., Monnig K.A., Ryan E.T., Jang S., Liang D.Yu, Liang M.S. Materials Research Society Symposium Proceeding, 2002, 686, A9.7.

21. Krause-Rehberg R., Leipner H.S. Positron annihilation in semiconductors Springer series in solid state sciences 127. Springer Verlag, Berlin 1999.

22. Benosman N., Amrane N., Mecabih S., Aourag H., J. Phys. and Chem. Of Solids, 2000, 61, 1727-1733.

23. Amrane Na., Soudini B., Amrane N., Aourag H., Mater. Sci. and Eng. B, 1996, 40, 119-125.

24. Puska M.J., Nieminen R.M., Reviews of Modern Physics, 1994, 66, 841.

25. Nabi Z., Abbar B., Amrane N., Aourag H., Mater. Chem. And Phys., 1999, 60, 256-261. 
26. Kohn W., Sham K., Phys. Rev. A, 1965, 140, 1133-1138.

27. Perdew J.P., Burke K., Ernzerhof M., Phys. Rev. Lett., 1996, 77, 3865-3868.

28. Liming W., Panda B.K., Fung S., Beiling C.D., J. Phys: Cond. Matter, 1997, 9, 8147-8154.

29. Leguey T., Monge M.A., Pareja R., Riveiro J.M., J. Phys: Cond. Matter, 1995, 7, 6179-6185.

30. Chelikowsky J.R, Cohen M.L., Phys. Rev. B., 1976, 14, 2.

31. Friedel P., Hybertsen M.S., Schlüter M., Phys. Rev. B., 1989, 39, 7974-7977.

32. Deibuk V.G., Viklyuk Ya.I., Rarenko I.M., Semiconductors, 1999, 33, No. 3, 293-296.

33. Jarlborg T., Manuel A.A., Peter M., Sanchez D., Singh A.K., Stephan J.-L., Walker E. Positron Annihilation (eds. L.Dorikens-Vanpraet, M.Dorikens and D.Segers). World Scientific, Singapore, 266, 1988.

34. Corbel C., Stucky M., Hautojarvi P., Saarinen K., Moser P., Phys. Rev. B, 1988, 53, 8192.

35. Lock D.G., Crisp V.H.C., West R.N., J. Phys. F, 1973, 3, 561.

36. M.L.Cohen, T.K.Bergstresser, Phys. Rev., 1966, 141, 789.

37. Phillips J.C., J. Phys. Soc. Japan, 1966, 21 (Supp.), 3.

38. Aourag H., Khelifa B., Belaidi A., Tadjer A., Rezki M., Gamoudi M., Phys. Stat. Sol. (b), 1990, 160, 193.

39. Fujiwara K., Hyodo T., J. Phys. Soc. Jpn, 1973, 35, 1133.

40. Saito M., Oshiyama A., Tanigawa S., Private communication.

41. Liu W., Berko S., Mills A.P. Jr. Positron annihilation, Matter., 1992, Sci. Forum, Szombachely, 743.

42. Tsay Y.F., Vaidyanathan A., Mitra M.S., Phys. Rev. B, 1979, 19, 5422.

\title{
Позитронна анігіляція в нітриді бору
}

\author{
Н.Амране \\ Університет Об'єднаних Арабських Еміратів, природознавчий факультет, Об'єднані Арабські \\ Емірати \\ Отримано 24 січня 2006 р., в остаточному вигляді - 20 травня 2006 р. \\ Електронні і позитронні зарядові густини є обчислені як функції положення одиничної комірки для \\ нітриду бору. Хвильові функції отримані, відповідно, з обчислень псевдопотенціальної зонної стру- \\ ктури і наближених незалежних частинок (IPM) для електронів і позитронів. Спостережено, що по- \\ зитронна густина є максимальною у відкритих щілинах, і є обмеженою не тільки іонним кором, але \\ також, значною мірою, валентними зонами. Густини електрон-позитронного моменту обчислені для \\ площин $(001,110)$. Результати використані для аналізу позитронних ефектів у BN.
}

Ключові слова: позитронна анігіляція, густина імпульсу, кутова кореляція

PACS: $78.70 . B j, 71.15 . D x$ 